Title:

\title{
Live demonstration: A VCO-based point-of-care ESR spectrometer
}

Author(s): Schlecker, B., Chu, A., Handwerker, J., Kunstner, S., Ortmanns, M., Lips, K., \& Anders, J.

Document type: Postprint

Terms of Use: $\quad$ Copyright applies. A non-exclusive, non-transferable and limited right to use is granted. This document is intended solely for personal, non-commercial use.

Citation:

(C) 2017 IEEE. Personal use of this material is permitted. Permission from IEEE must be obtained for all other uses, in any current or future media, including reprinting/republishing this material for advertising or promotional purposes, creating new collective works, for resale or redistribution to servers or lists, or reuse of any copyrighted component of this work in other works.

Schlecker, B., Chu, A., Handwerker, J., Kunstner, S., Ortmanns, M., Lips, K., \& Anders, J. (2017). Live demonstration: A VCO-based point-of-care ESR spectrometer. 2017 IEEE SENSORS. https://doi.org/10.1109/icsens.2017.8234031 


\title{
Live Demonstration: A VCO-based point-of-care ESR spectrometer
}

\author{
B. Schlecker ${ }^{1}$, A. Chu ${ }^{1}$, J. Handwerker ${ }^{1}$, S. Künstner ${ }^{2}$, M. Ortmanns ${ }^{1}$, K. Lips ${ }^{2}$ and J. Anders ${ }^{1}$ \\ Email: jens.anders@uni-ulm.de \\ ${ }^{1}$ Institute of Microelectronics, University of Ulm, D-89081 Ulm, Germany \\ ${ }^{2}$ Berlin Joint EPR Lab, Helmholtz Zentrum Berlin für Materialien und Energie, Germany
}

\section{INTRODUCTION}

Thanks to its unmatched specificity, electron spin resonance (ESR) spectroscopy is amongst the most powerful analytical techniques today with applications ranging from the life sciences over material science analysis to food quality control [1]. By measuring the spin of an unpaired electron, ESR spectroscopy is specifically suited to analyze free radicals, which play a major role in many diseases and can also directly be related to premature cell aging.

In contrast to the related and more widely known technique of nuclear magnetic resonance (NMR) spectroscopy, ESR electronics operate at significantly higher frequencies which in combination with the very short relaxation times of electrons is the reason why today most ESR experiments are still performed in the continuous-wave $(\mathrm{cw})$ mode. In a conventional cwESR experiment, an ESR sample is placed inside a mechanically tunable resonator which in turn is placed in a sweepable, strong static magnetic field $B_{0}$ and the sample is irradiated with a second magnetic field $B_{1}$, whose frequency satisfies the resonant condition $\omega_{\text {osc }}=\gamma B_{0}$, where $\gamma \approx 2 \pi \cdot 28 \mathrm{GHz} / \mathrm{T}$ is the so-called gyromagnetic ratio of an electron. In conventional setups, the sweepable magnet is realized as a bulky, expensive and power-hungry electromagnet and the $B_{1}$-field for the excitation is generated by microwave source, whose output is connected to the resonator containing the sample using expensive microwave components (waveguides, circulator).

\section{DEMONSTRATED SYSTEM: BATTERY OPERATED, VCO-BASED ESR-ON-A-CHIP}

In the proposed live demo, we will present an improved version of our portable, battery-operated, point-of-care ESR spectrometer, which was previously presented at ISSCC [2]. At its heart, our system uses a VCO-based ESR detector, which removes many of the abovementioned limitations of conventional ESR setups. The system consists of an inexpensive, donut size, highly homogeneous permanent magnet (Metrolab PM 1055) as source of the static $B_{0}$-field and a custom ASIC with an integrated CMOS LC-tank VCO, which works both as $B_{1}$-field source to excite the spin transitions and which at the same time detects the ESR signal as changes of its oscillation frequency. The architecture of the presented ESR spectrometer with its individual building blocks is shown in Fig. 1. Here, compared to a conventional ESR system, the ESR spectrum

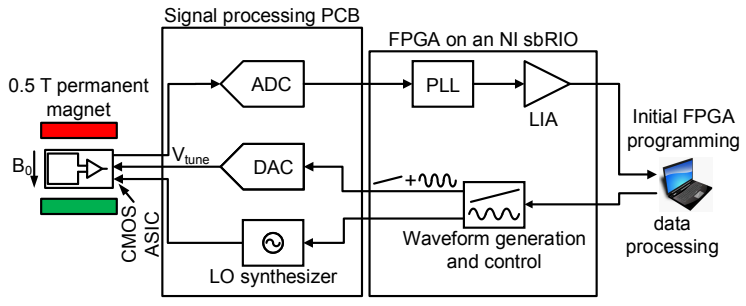

Fig. 1: Presented VCO-based ESR PoC setup proposed in [2].

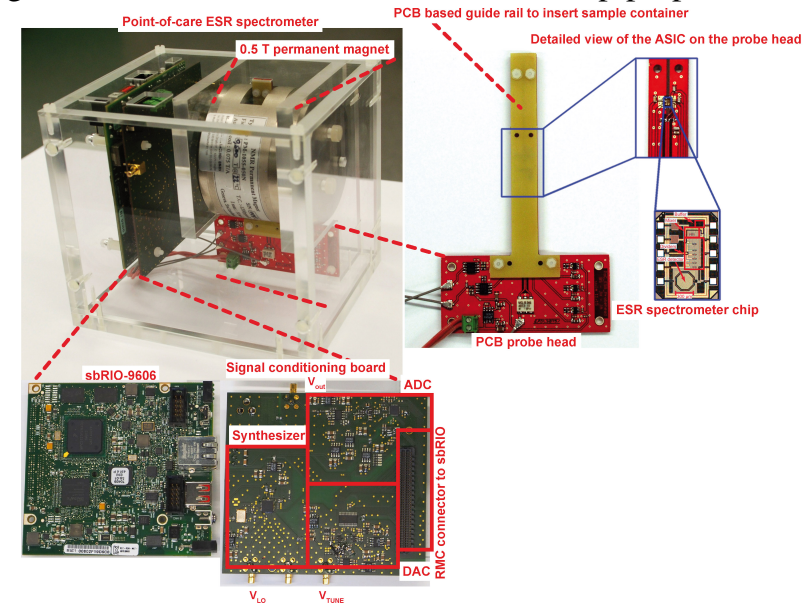

Fig. 2: Annotated photograph of the VCO-based ESR PoC setup presented in [2].

is recorded by means of a frequency sweep instead of a field sweep, which removes the need for a bulky and expensive electromagnet. Fig. 2 then displays an annotated photograph of the assembled portable spectrometer with detail views of its individual components.

As a novelty compare to the system presented in [2], we present a novel VCO tuning scheme, which greatly relaxes the requirements on the digital-to-analog-converter (DAC) driving the VCO tuning ports.

\section{REFERENCES}

[1] U. T. Twahir, C. N. Stedwell, C. T. Lee, N. G. J. Richards, N. C. Polfer, and A. Angerhofer, "Observation of superoxide production during catalysis of bacillus subtilis oxalate decarboxylase at ph 4," Free Radical Biology and Medicine, vol. 80, pp. 59-66, 2015.

[2] J. Handwerker, B. Schlecker, U. Wachter, P. Radermacher, M. Ortmanns, and J. Anders, "A 14ghz battery-operated point-of-care esr spectrometer based on a $0.13 \mu \mathrm{m}$ cmos asic," in 2016 IEEE Int. Solid-State Circ. Conf. (ISSCC), Jan 2016, pp. 476-477. DOI: 10.1109/ISSCC.2016.7418114 